\title{
Decision Supporting System in Agriculture through IOT
}

\author{
M.Sowjanya ${ }^{1}$, D. Leela Dharani ${ }^{2}$ \\ ${ }^{1,2}$ Asst Professor, Dept of IT, PVP Siddhartha Institute of Technology, Vijayawada. \\ ${ }^{1}$ sowjanya@pvpsiddhartha.ac.in
}

\begin{abstract}
As India is an agriculture background country, preserving the soil nutrients is essential. Agriculture is a major stream that helps us to meet the growing need for food in the country. Since agriculture is highly dependent on the soil, maintenance of soil minerals is necessary to reduce the drop in the crop yield. To achieve this, the easiest way is by applying Fertilizers. Measurement of soil Nitrogen $(\mathrm{N})$, Phosphorus $(\mathrm{P})$ and Potassium $(\mathrm{K})$ is necessary to determine the suitable Fertilizer. The work here designs an NPK sensor with Light Dependent Resistor (LDR) and Light Emitting Diodes to measure the NPK mineral values present in the soil. The data sensed by the designed NPK sensor from the selected agricultural fields is sent to the cloud using Thingspeak tool. A database is created by recording LDR data and NPK mineral values. On this database logistic regression algorithm is applied and the machine learning model is trained. The predicted data from the Machine learning model is now saved for future reference. The Results of the analysis are used for predicting the suitable fertilizer from the Fertilizer database and then the suggestions are sent to the farmer's device using GSM module. This way the Soil testing is done effectively and the results are sent to the farmer much faster than most of the soil tests that are available today.
\end{abstract}

Key words: LDR, NPK, IOT.

\section{INTRODUCTION}

Agriculture is most widely done in many places and in many countries. This is major occupation of many countries to contribute to solve the issues for food scarcity. This occupation of more success if the farmers produced high yield in their cultivation. If the cultivation is low, this will affect the country economy. In many countries if there is low cultivation, there is no proper use of fertilizers by the farmers. To overcome these issues the fertilizers can be added at better quantity because of the missing of various nutrition in the soil. Testing is to be done for the soil to add the more minerals to increase the cultivation in that selected soil. Based on the efforts of the farmer the effective cultivation can be done and gradually plant growth can be increased based on the soil testing.

Many of the developed countries encourage their farmers to increase the cultivation with the integration of the Internet of
Things (IoT). IoT is the networking components that will used to improve the cultivation. Various sensors are used to test the soil and these soil data is sent to the server with internet. IoT is fast growing system to increase the performance of the any type of application. In this paper, the IOT is integrated with the machine learning to increase the system with the help of a LDR sensor and Logistic Regression Algorithm to facilitate the farmers about the awareness of soil nutrients deficiency and appropriate fertilizer to be added to the fields at right time.

The objective of proposed work is to predict Mineral compositions in the soil and determining the suitable fertilizer to the soil sample.

A dataset is created by reading the LDR values of soil samples for which the mineral compositions are known. The dataset is then used to train a Machine learning model using Logistic regression Algorithm.

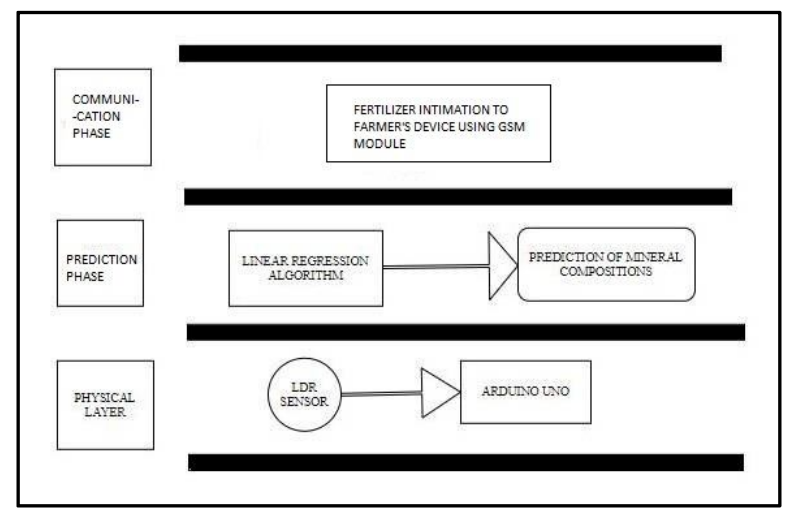

\section{LITERATURE SURVEY}

Cropping continuously without the adequate estimation and provisioning of the soil supplement may endanger the reasonability of the cultivation. Soil supplement estimation is altogether required for fitting plant improvement and feasible readiness. A key in soil testing for characterized treatment is to choose the proportion of soil supplements, trailed by proposition of the enhancement [1] needs and site unequivocal planning. Nitrogen, Phosphorus and Potassium are the three huge enhancements required for the plant advancement. In the present work electrochemical sensor has been made to choose the $\mathrm{N}, \mathrm{P}, \mathrm{K}$ and various types of enhancements present in the soil. 
Progression of cultivation using advancement will be a great deal of significant being developed. For another rustic zone, without knowing or checking the critical parameters of the earth, improvement will be problematic hence the farmers suffer [4] cash related disasters. This errand gives a short graph of the soil checking structure using sensors. Distinctive soil sensors are used to check temperature, sogginess and light, moisture and ph regard. The information from the sensors in the earth is [5] sent to the MCP3204 A/D converter then from $\mathrm{A} / \mathrm{D}$ converter it send to the cloud through Raspberry pi. Finally we can see the information saved to cloud on mobile phone similarly as PC. In view of information we understand which yield is sensible with given soil parameter. Consequently this pattern setting development urges the farmers to know the exact parameters of the earth along these lines making the soil testing approach less complex.

\section{RESULTS AND OBSERVATIONS}

\section{Test case Results}

The required packages are NumPy and pandas are imported using the import statement. NumPy package is used to gain high end array functions and pandas is used for data frame functions. The warnings package is also imported so that no warnings are displayed. The csv file is imported using read_csv function in pandas package into nitrogen data.

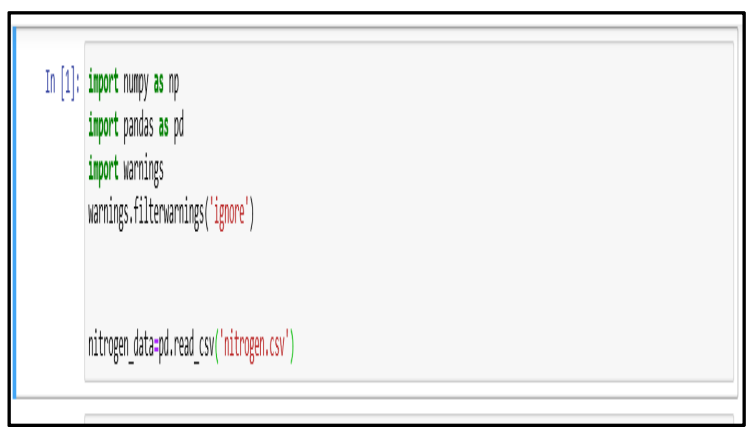

Figure 1: Nitrogen test_data input to regression model

The values in the data framenitrogen_data are displayed using the head () function in Python. This displays the values stored in data frame as a table.

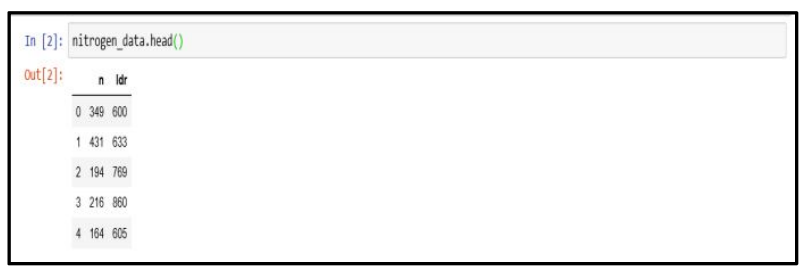

Figure 2: Sample data frame view using head()
The number of rows and columns are known by using the shape () function. The number of rows and columns of nitrogen_data are known using this function.

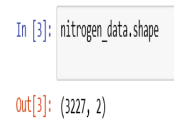

Figure 3: To display size of dataset using "shape" function.

The dataframe is tested for any presence of null values using is null () function.

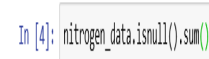

$$
\begin{aligned}
& \text { at[4]: } 1 \\
& \text { Idr } \theta \\
& \text { dtype: intol }
\end{aligned}
$$

Figure 4: isnull() function to identify null values

This is the model used in data mining. The data that is preprocessed is now split into two parts namelyTraining Data, Test Data.

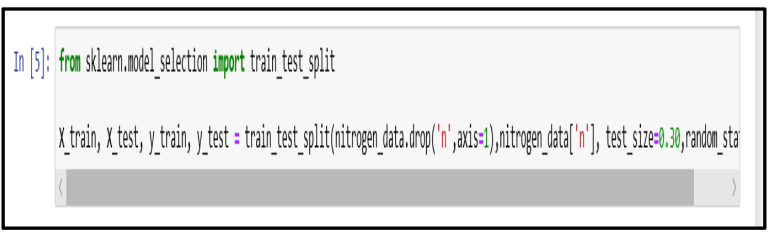

Figure 5: dataset divided into x_train\&x_test in 7:3

The training data is split accordingly so that the data is not under fitted or over fitted. Under fitted means low amount of data is taken for training and the model is not perfect for prediction. Over fitted means high amount of data is taken for training and the model cannot verified over a small amount ofdata for testing. The data must be split accordingly such that it's neither under fitted nor over fitted. In our case, data is split into $70 \%$ as training data and $30 \%$ as testing data. This train test split is done by using the train_test_split module in sklearn package. The train_test_split module consists of function namely train_test_split () which does all the work mentioned above.The size that the data needs to be split is mentioned by us and a random state is used so that different types of data are not formed for every execution. The training data is further processed such that a model is ready for testing.

All the packages that are useful for logistic regression model are imported and a logistic regression model is built on the training data (x_train, y_train) and tested for accuracy later 


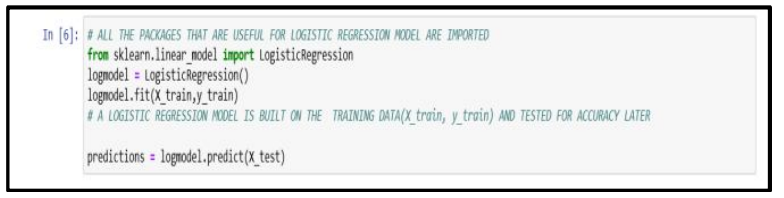

Figure 6: Logistic Regression model trained using x_train set

The predictions made in the above part of the code are printed. The values of the nitrogen that are predicted will be known

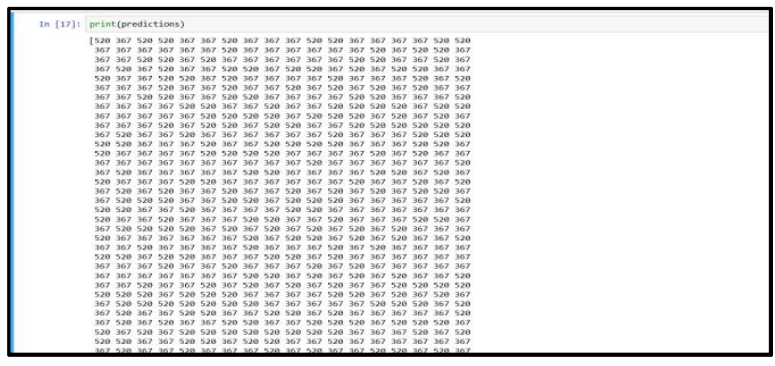

Figure 7: Above picture depicts the predicted Nitrogen values

The train and test data is studied and output is stored as nitrogen_output

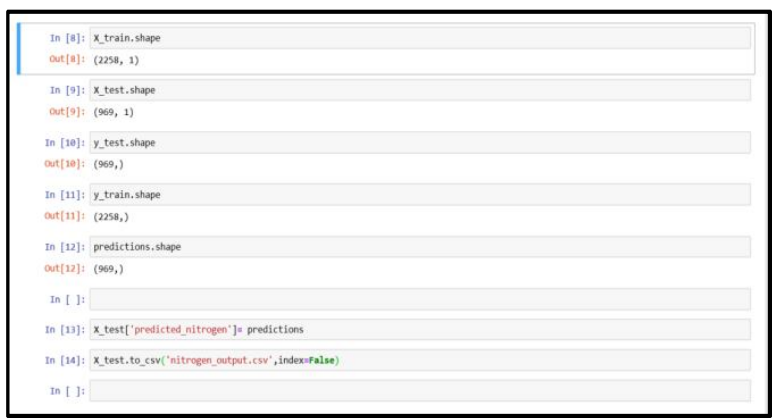

Figure 8: The predictions are then saved in "nitrogen_output.csv"

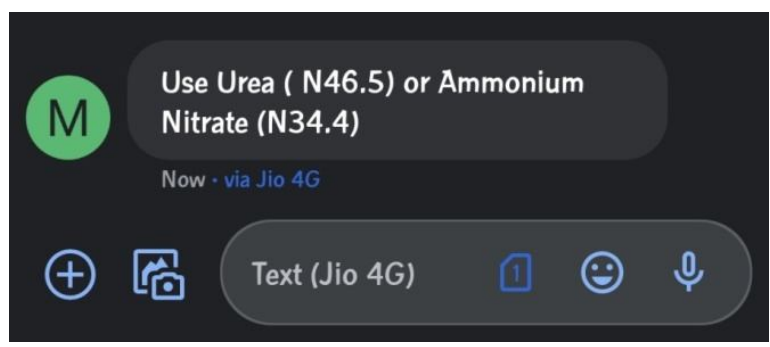

Figure 9: Fertilizer intimation to farmer/user for low Nitrogen using GSM module

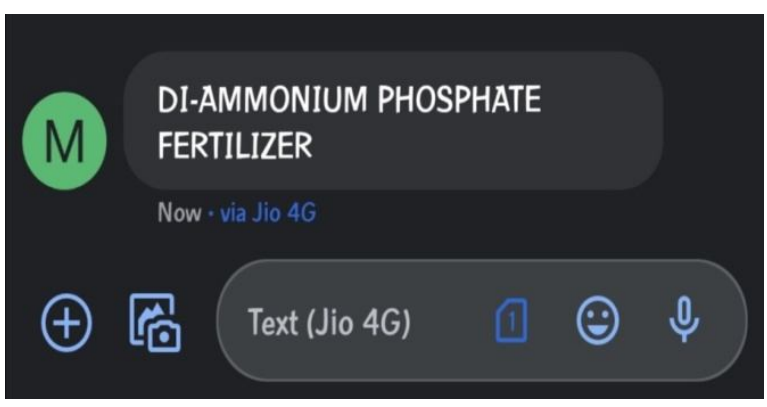

Figure 10: Fertilizer intimation to user/farmer for low Phosphorus using GSM module

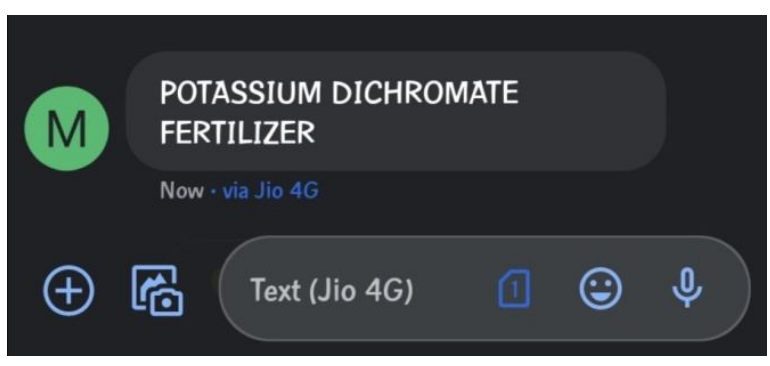

Figure 11: Fertilizer intimation to user/farmer for low potassium using GSM module

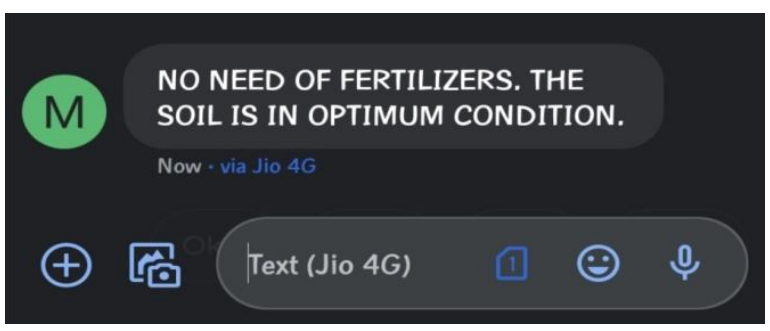

Figure 12: Response from the GSM module for a nearly ideal sample

\section{Observations}

The results of this system, using LDR sensor module and Machine learning, helps detect Minerals in soil which helps in providing better chances for choosing the appropriate fertilizer to use. Although it is still far from very good performance, this model is better for use since it is able to predict the fertilizers instantly and intimate the farmer/Client which fertilizers are needed to the crops to reduce loss and provide with a maximum yield.

\section{CONCLUSION}

Testing of soils to check the nutrients in the soil will help the increase the cultivation. In this paper, the proposed system focus on provides the information about the soil to the farmer regarding the unavailability of the macro-nutrients namely nitrogen, phosphorous and potassium through SMS using the designed NPK sensor. Thus the integration of IOT is very low cost provides the better cultivation 


\section{REFERENCES}

1. Luis Ruiz-Garcia, LoredanaLanadei, The role of RFID in agriculture, applications, limitations \& changes, J. Comput. Electron. Agric. (2017) 42-50

2. TamoghnaOjha, SudipMisra, Narendra, WSN for agriculture: state of the art in practice and future challenges, J. Comput. Electron. Agric. (2015) 66-84.

3. E. Ben-Dor, A. Banin, Near infrared analysis as a rapid method to simultaneously evaluate several soil properties, Soil Sci. Soc. Am. J. (1993)364-372.

4. W. Van Lierop, Determination of available phosphorus in acid and calcareous soils with the Kelowna multipleelement extractant, Soil Sci. (1988) 284-291.

5. D.R. Keeney, J.M. Bremner, Comparison and evaluation of laboratory methods of obtaining an index of soil nitrogen availability, Agron. J. (1966) 498-503.

6. J.C. Ezeokonkwo, Engineering properties of NPK fertilizer modified Soil, J.Emerg Trends Eng. Appl. Sci. (2011) 962-966.

7. Allen Doyle, Michael N. Weintraub, Joshua P. Schimel, Persulfate digestion and simultaneous colorimetric analysis of carbon and nitrogen in soil extracts, Soil Sci. Soc. Am. J. (2002) 669-676.

8. Yong He, Kai Dong, Yongheng $\mathrm{Hu}$, Tao Dong, Colorimetric recognition for urinalysis dipsticks based on quadratic discriminant analysis, in: 39th Annual International Conference of the IEEE - Engineering in Medicine and Biology Society, 2017

9. Henriksen, A.R. Selmer-Olsen, Automatic methods for determining nitrate and nitrite in water and soil extracts, Analyst (1970) 514-518.

10. Rigor G. Regalado, Jenefer C. Dela Cruz, Soil pH and nutrient (nitrogen, phosphorus and potassium) analyzer using colorimetry, IEEE TENCON (2016).

11. Deepa V. Ramane, Supriya S. Patel, A.D. Shaligram, Detection of NPK nutrients of soil using fiber optic sensor, Int. J. Res. Advent Technol. (2015) 66-70.

12. Dissanayake H. Pasqual, B.C.L. Athapattu, Economical colorimetric smart sensor to measure water quality of drinking water in CKDu prevalence areas, IEEE Sens. J. (2017) 5885-5891.

13. Yu Chen, Guoqing Fu, Yael Zilberman, WeitongRuan, ShidehKabiriAmeri, Eric Miller, Sameer Sonkusale, Disposable colorimetric geometric barcode sensor for food quality monitoring, 19th International Conference on Solid-State Sensors, Actuators and Microsystems (TRANSDUCERS) (2017).

14. Dami Kim, Sejin Kim, Jeongho An, Sanghyo Kim, A portable colorimetric array reader for toxic gas detection, ISOCS/IEEE International Symposium on Olfaction and Electronic Nose (ISOEN) (2017).

15. Cheng-Shane Chu, Meng-Wei Hsieh, Optical carbon dioxide sensor based on the colorimetric change of naphtholphthalein and internal reference fluorescent CIS/ZnS QDs, 25th Optical Fiber Sensors Conference (OFS) (2017).

16. Abdul Kodir, CukImawan, IndraSetiaPermana, WindriHandayani, Pesticide colorimetric sensor based on silver nanoparticles modified by L-cysteine, International Seminar on Sensors, Instrumentation, Measurement and Metrology (ISSIMM) (2017).

17. A. Zakarian, Analysis of process models: a fuzzy logic approach, Int. J. Adv.Manuf. Technol. (2001) 444-452. 